\title{
Strategic behavior without awareness? Effects of implicit learning in the Eriksen flanker paradigm
}

\author{
Rodica GHINESCU \\ Lincoln University, Jefferson City, Missouri \\ Todd R. Schachtman and Michael A. Stadler \\ University of Missouri, Columbia, Missouri \\ AND \\ Monica Fabiani AND Gabriele Gratton \\ University of Illinois at Urbana-Champaign, Urbana, Illinois
}

\begin{abstract}
This experiment investigated whether subjects' selection and use of strategies in detecting a target letter in a flanker task requires intention. Subjects' expectancies for compatible and incompatible trials (trials on which the response to the flanker stimulus was consistent or inconsistent with the target response) were manipulated by presenting cues that signaled the occurrence of these types of trials. Three groups of subjects received explicit, partially explicit, or implicit instructions about the meaning of the cues. By the end of the experiment, all the groups were able to select and use strategies based on the cues to improve their performance. However, this strategy selection developed slowly with practice in the latter two groups, whereas it was present from the outset in the first group. In addition, forced choice tests performed after the experiment showed that the subjects in the implicit condition could not intentionally indicate which stimuli were most likely to follow a given cue. Thus, the data suggest that the selection of strategies occurred outside the subjects' awareness, and without their intention.
\end{abstract}

All cognitive tasks probably involve at least some minimal strategy selection. When people are required repeatedly to choose among different possibilities and to analyze the costs and benefits of a given situation, they learn rules and develop strategies to perform the task. Although in many cases the selection of a particular strategy is a deliberate process, in other instances it may also involve mechanisms that are not under conscious awareness. The present study investigates conditions in which both types of strategy selection may occur.

Numerous studies have demonstrated that even under time pressure, subjects are able to extract relevant information from the environment and use strategies to direct their task performance (e.g., Coles, Gratton, Bashore, Eriksen, \& Donchin, 1985; Gratton, Coles, Sirevaag, Eriksen, \& Donchin, 1988; Logan \& Zbrodoff, 1979, 1982; Szymanski \& MacLeod, 1996). Gratton, Coles, and Donchin (1992), using the Eriksen flanker task (Eriksen \& Eriksen, 1974), showed that strategy selection is adaptive and depends on expectations about stimulus conditions. Specifically, when subjects were led to expect that compatible-noise conditions (i.e., conditions in which target and noise letters were associated with the same re- sponse) would occur, they used a strategy leading to fast responses, but with high susceptibility to distractor conditions. When subjects were led to expect that incompatiblenoise conditions (i.e., conditions in which target and noise letters were associated with conflicting responses) would occur, they used a strategy leading to slow responses, but also to low susceptibility to distractor conditions.

Gratton et al. (1992) did not investigate the extent to which these forms of strategy adjustments require that the subjects have explicit awareness of the difference in noise expectancy conditions (i.e., compatible vs. incompatible trials). In fact, evidence from event-related brain potential (ERP) studies (e.g., mismatch negativity; see Näätänen, 1982) and investigations of implicit-learning processes (e.g., Carlson \& Flowers, 1996; Miller, 1987; Stadler, Warren, \& Lesch, 2000) suggest that subjects are able to compute probabilities of events even in the apparent absence of awareness. If expectancies can be computed at a subconscious level, can strategic choices made on the basis of expectancy manipulations also occur without explicit awareness? The present study examined this possibility.

As in Gratton et al. (1992), the subjects in the present study were presented with a visual display (the imperative

T. R. Schachtman, schachtmant@missouri.edu 
stimulus) composed of five letters (HHHHH, SSHSS, SSSSS, and HHSHH). The middle letter was designated as the target, and the other letters (labeled noise letters) were used as distractors. The subjects were instructed to respond to one of the possible target letters $(\mathrm{H}$ or $\mathrm{S})$ with one hand and to the other letter with the other hand. Thus, two conditions were created: one in which the target letter had the same response as the noise letters (HHHHH or SSSSS; compatible-noise condition) and another in which the noise letters were associated with the alternative response (HHSHH or SSHSS; incompatible-noise condition). In this situation, the incompatible-noise condition leads to slower responses and larger error rates than does the compatible condition (Eriksen \& Eriksen, 1974). The difference in reaction times (RTs) between the two conditions is usually attributed to involuntary processing of the noise letters and to response competition from automatically activated responses. If the incorrect response is activated by the noise letters being processed on an incompatible trial, this will compete with the correct response, and thus a longer time will be needed to initiate the overt response and more errors will occur on such trials. These effects of the noise are called the flanker effect.

On the basis of findings like Gratton et al.'s (1992) in which subjects' expectancies for compatible or incompatible trials could be manipulated by presenting cues that predict the type of upcoming trial, Kunde (2003) suggested that theoretically automatic response activation processes were not, in fact, completely automatic. Instead, he proposed that the response activation process could be gated, such that contextual factors that were predictive of potential response conflict could modulate the automatic activation process. When primes were more likely to facilitate responding, the automatic process would be allowed to operate. However, when the primes were more likely to cause response conflict, the automatic process would be gated to prevent errors. This distinction is similar to the distinction that Gratton et al. (1992) made between parallel and focused strategies, respectively. In the former, subjects adopt a wide attentional focus, whereas in the latter, the attentional focus is narrower.

To investigate the nature of his hypothesized gating process, Kunde (2003) presented discriminable and nondiscriminable primes before the presentation of an imperative stimulus and found that the gating process, due to sequential effects (i.e., to the incongruence between the prime and the target on the preceding trial), appeared to occur only when subjects were aware of the prime (i.e., only in the discriminable condition). He argued that intention is needed for this type of gating process (which may be labeled as a reactive control process). However, it is likely that the information conveyed by the nondiscriminable primes in Kunde's (2003) study was not available to the hypothesized gating mechanism, because those primes were presented only for $14 \mathrm{msec}$, although it clearly interfered with the processing of the current stimulus, generating a large incongruence effect. Interestingly, there was no evidence that these very briefly presented primes could be consciously recognized by subjects: A discrimination test showed that the subjects could not reliably distinguish one prime from another at this presentation rate (however, see Kunde, 2004, and Kunde \& Wühr, 2006, for a demonstration of a different type of prime exerting an effect on RT performance). Kunde and Wühr also manipulated prime discriminability, as Kunde (2003) did, and found that longer prime exposure time resulted in larger conflict effects. However, they confounded the prime-target onset time with prime duration. Thus, it may have been the amount of time available for stimulus processing, conscious or unconscious, rather than awareness of the prime, that influenced performance.

The data by Kunde and collaborators raise the possibility that the level of awareness of a particular stimulus may be critical in determining the extent to which it may trigger adaptive control processes. In Kunde's work, it is awareness of the conflict that is investigated; here, we are interested in awareness of the contingencies triggering the adaptive control event itself. Kunde and collaborators did not directly investigate this issue. In this article, we specifically address the question of whether explicit knowledge of the relationship between the inducing event (in our case, a supraliminal cue) and the type of noise in the imperative stimulus is critical to the adaptive process.

Since an objective definition of subliminal processing is somewhat complex (e.g., Cheesman \& Merikle, 1984; Reingold \& Merikle, 1988), in the present research we sidestepped the issue by using an implicit learning procedure instead of a subliminal perception procedure. A suprathreshold cue stimulus (the letter A, B, or C) was presented before the imperative stimulus. We established contingencies between particular cues and the probability of occurrence of a compatible or incompatible trial. On the basis of Gratton et al. (1992), it was expected that, at least when subjects were aware of the contingencies, they would use the information given by the cues to improve their performance and would be able to vary their strategies as a function of the cue. In order to explore the possibility that strategy selection might also occur implicitly, the subjects were divided into three groups and received explicit, partially explicit, or implicit instructions regarding the predictive value of the cues. If strategy selection can be learned implicitly as well as explicitly, the instruction type should not influence the size of strategic adjustments made by the subjects; if, instead, strategy selection requires explicit knowledge, the instruction type should have an effect. If expectancies can be computed at a subconscious level, can strategic choices made on the basis of those expectancies also occur at a subconscious level (i.e., without awareness)? Such a finding would suggest that Kunde's (2003) hypothesized gating process can operate outside of awareness. However, under implicit instructions, the relationship between the cues and the noise probability can be learned only over time. Therefore, we separated trials occurring at different times during the experiment to assess whether gradual learning of the cuestimulus contingency (i.e., strategic adjustments) in fact occurred. 


\section{METHOD}

\section{Subjects}

Eighty-two students ( 38 women and 44 men) at the University of Missouri-Columbia received course credit for their participation in this experiment. The subjects were $18-27$ years old and had normal or corrected-to-normal vision and hearing. Each subject was tested individually and completed a three-part experiment. The first part was devoted to practice and involved 5 blocks of 30 trials; in the second part, the subjects completed 64 blocks of 30 trials; in the third and final part, the subjects completed paper-and-pencil tests. All the subjects gave informed, written consent prior to testing.

\section{Stimuli}

The stimuli were presented on a computer screen. Two stimuli were presented on each trial. The subjects sat approximately $1 \mathrm{~m}$ from the computer monitor, with their eyes level and fixated on the screen's center. The first (cue) stimulus was a letter (A, B, or C) and subtended a visual angle of $0.5^{\circ}$. The second (imperative) stimulus consisted of one of four 5-letter arrays (HHHHH, SSHSS, SSSSS, or HHSHH). The visual angle subtended by each letter in the array was approximately $0.5^{\circ}$. The angle subtended by the whole array was approximately $2.5^{\circ}$.

The cues had predictive values. A indicated, with a .8 probability, that the upcoming imperative stimulus would be compatible, and $\mathrm{C}$ indicated, with a .8 probability, that it would be incompatible. Cue B indicated, with a .5 probability, that the upcoming imperative stimulus was equally likely to be compatible or incompatible. The actual letters used as cues (A, B, C) were counterbalanced across subjects with respect to the predictive role they possessed, but we will use the combinations just described as our example throughout this article. To minimize visual search, a fixation cross was placed at the center of the computer screen, $0.1^{\circ}$ below the location of the target letter, and remained constantly visible during the experiment. On each trial, the cue was presented randomly $1^{\circ}$ to the left or right side of the fixation cross for $100 \mathrm{msec}$, and it was followed by the imperative stimulus after $1,500 \mathrm{msec}$. The imperative stimulus (letter array) was presented for $100 \mathrm{msec}$. The time allocated for responding was $1,500 \mathrm{msec}$. If no response occurred within that period, no data were recorded for that trial.

The cues were randomly ordered within each block of trials, with each of the cues occurring on $33.3 \%$ of the trials. The overall probability for both compatible- and incompatible-noise trials was .5 within each block.

\section{Procedure}

The subjects were divided into three equal-size groups and received different instructions. At the beginning of the experiment, the subjects in the first group (explicit instructions) were informed as to what the cues predicted (with respect to the probability of the trial type) and were told to use this information when responding. In particular, they were asked to respond to their first overall impression of the stimulus array when they expected a compatible trial (parallel strategy), to focus on the central letter when an incompatible trial was expected (focused strategy), and to use any strategy when a compatible and an incompatible trial were equally likely.

The subjects in the second group (partially explicit instructions) were informed only that the cues had some predictive value. Specifically, they were told that the cues predicted whether the upcoming array would be compatible or incompatible. No information about the probabilistic values of the cues was given. The subjects were told that by being exposed to many trials, they should be able to predict what kind of condition would occur on the basis of the cue, and, consequently, they should be able to use this information when responding. Like the first group, they were instructed to respond to their first overall impression of the stimulus array when they expected a compatible trial, to focus on the central letter when an incompatible trial was expected, and to use any strategy when a compatible and an incompatible trial were equally likely. The third group (implicit instructions) received no information about the meaning of the cues. The subjects in this group were told that a symbol would precede each occurrence of a stimulus array and that their task was to respond to the central letter of the stimulus array.

All the subjects were instructed to respond to the central letter of the array by pressing one of the two keys on a response box. They were asked to respond with one hand if the central letter was $\mathrm{H}$ and with the other hand if the central letter was S. The letter-hand association was counterbalanced across subjects. During the practice blocks, the subjects were instructed to respond as quickly as possible without making more than $10 \%-20 \%$ errors per block and were asked between blocks to respond more quickly if their error rate fell below $10 \%$.

To determine whether learning of the contingency between cues and imperative stimuli did in fact occur, trials occurring at different times during the experiment were sorted into three groups: early trials (first $25 \%$ of the trials), middle trials (middle $50 \%$ of the trials), and late trials (last $25 \%$ of the trials).

After the experiment, all the subjects were asked to complete two questionnaires. The first questionnaire was a forced choice task. Each subject received a list that was made up by combining each of the cues with a pair of arrays. The arrays in each pair were various combinations of two of the possible four arrays used as imperative stimuli in the study. There were six such combinations (HHHHH with SSSSS, HHSHH or SSHSS; SSSSS with either SSHSS or HHSHH; and HHSHH with SSHSS). Since these were combined with each of the cues, there were a total of 18 trials, which were presented in random order. The subjects were asked to circle which of the two arrays was more likely to follow the cue. Note that the conditions in which the subjects had to select between two compatible or two incompatible arrays were not used for the analyses. However, no subject reported problems in providing answers for these trials.

The second questionnaire was a probability estimation task. Twelve stimuli were created, with each cue followed by one of the four possible arrays of letters. The subjects were asked to mark on a scale from 0 to 100 what percentage of the time a given cue was followed by a given array. The order of the forced choice and probability estimation tasks was counterbalanced across subjects.

\section{RESULTS}

The data collected during the practice blocks were not analyzed. Also, trials on which no response was given or for which the response was faster than $200 \mathrm{msec}$ (i.e., fast guesses) or for which the response occurred after $1,200 \mathrm{msec}$ (considered to be outliers) were not included in the analyses. Eighteen mean RTs were obtained for each subject: three phases of the experiment (early [first quarter], middle [second and third quarters], and late [fourth quarter]); three cues (predict compatible, predict neutral, and predict incompatible); and two noise levels (compatible and incompatible). The average RTs across subjects for all the conditions are presented in the top panels of Figure 1. The overall ANOVA included these three withinsubjects factors, as well as instruction/group (explicit, partial, and implicit) as the between-subjects factor. The results included a main effect of noise level $[F(1,79)=$ 535.49, $\left.M S_{\mathrm{e}}=1,582, p<.0001\right]$, with longer RTs for incompatible $(515 \mathrm{msec})$ than for compatible $(467 \mathrm{msec})$ trials. As was expected, however, this effect interacted with the prediction condition $\left[F(2,158)=9.32, M S_{\mathrm{e}}=378, p<\right.$ $.001]$, with a larger flanker effect for the predict-compatible condition $(52 \mathrm{msec})$ than for the neutral cue $(49 \mathrm{msec})$ and predict-incompatible $(42 \mathrm{msec})$ conditions. There was also an effect of task phase $\left[F(2,158)=7.26, M S_{\mathrm{e}}=7,102\right.$, 


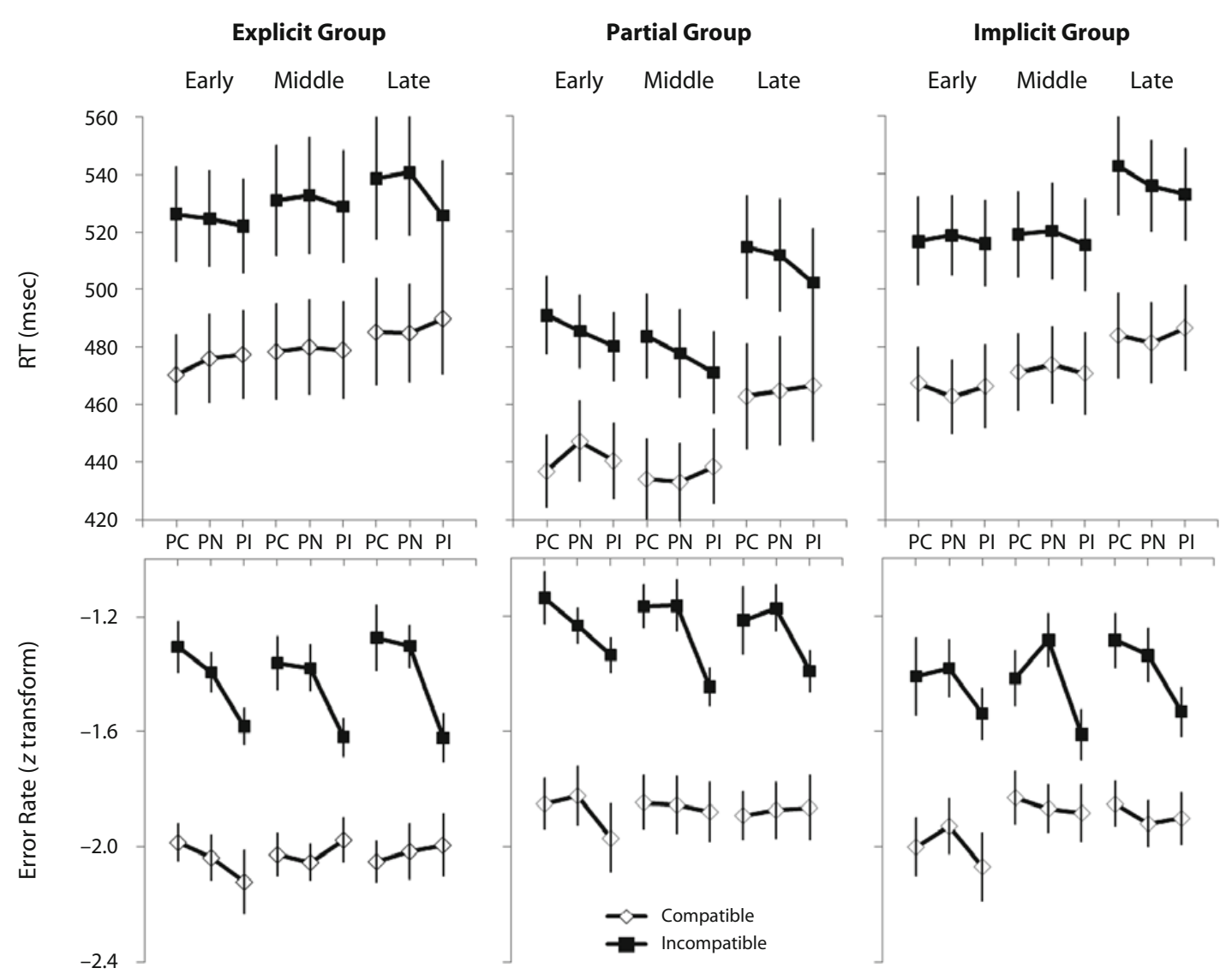

Figure 1. Mean reaction times (RTs; top) and error rates ( $z$ transform, bottom) for compatible (open diamonds) and incompatible (filled squares) trials preceded by predict-compatible (PC), predict-neutral (PN), and predict-incompatible (PI) cues. Data are represented separately for the early (first $25 \%$ of the trials), middle (intermediate $50 \%$ of the trials), and late (last $\mathbf{2 5 \%}$ of the trials) phases of the experiment and for each instruction group. Error bars refer to the standard errors of the means, computed across subjects.

$p<.001$ ], indicating a slowing down of the responses during the last quarter of the study $(506 \mathrm{msec})$ with respect to the first quarter $(485 \mathrm{msec})$ and the middle two quarters (486 msec), presumably as a result of fatigue.

Interestingly, instruction conditions did not result in any significant main effect or interaction. Specifically, and of particular importance for this article, there was no significant three-way interaction between instruction conditions, prediction cue condition, and noise level $[F(4,158)=$ $\left.0.94, M S_{\mathrm{e}}=378, \mathrm{n} . \mathrm{s}.\right]$, indicating that the modulation of the flanker effect determined by the predictive cue occurred similarly for all three instruction groups.

Since the interest of the study was on whether cue and instruction conditions would influence the size of the flanker effect, resulting in three- and four-way interactions that are difficult to extract from Figure 1, we present a portion of these data in Figure 2 in the form of differences between the RT (and accuracy) for compatible and incompatible arrays (the flanker effect), for each prediction and instruction condition. These data are, of course, consistent with the analyses and information presented above. To determine whether cues influenced performance in the implicit condi- tion, we calculated the difference in the flanker effect for the predict-compatible and predict-incompatible conditions during the last phase of the experiment for the subjects in the implicit group. Our prediction was directional: The flanker effect should be larger in the predict-compatible than in the predict-incompatible condition. A marginally significant effect was observed $[t(26)=1.56, p<.10$, one-tailed $]$.

These results indicate that the cue was used to adjust the subjects' strategies in a manner similar to that described in Gratton et al. (1992): When the subjects expected incompatible noise, they used a strategy that resulted in a smaller flanker effect than in the other conditions.

These data indicate that instructions (explicit, partially explicit, or implicit) made little difference in terms of the utilization of the cues for strategic adjustments. This was particularly evident by the end of the experiment. After a sufficient number of trials, all the groups of subjects, regardless of the types of instructions they received, were able to use the cue information to select an optimal strategy to perform the task.

The analysis of the accuracy data was conducted in a manner similar to that described for RTs. For these analy- 

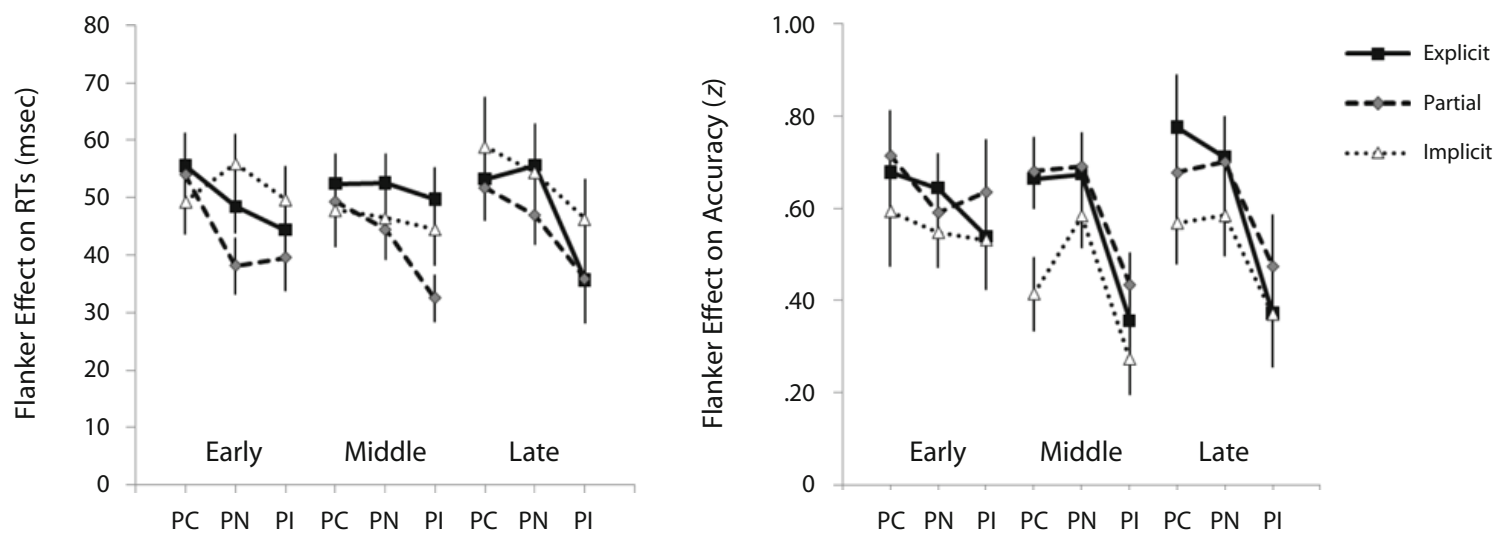

Figure 2. Flanker effects (difference between incompatible and compatible conditions) for RTs (left) and error rates ( $z$ transform, right) for trials preceded by predict-compatible (PC), predict-neutral (PN), and predict-incompatible (PI) cues. Data are represented separately for the early (first $25 \%$ of the trials), middle (intermediate $50 \%$ of the trials), and late (last $25 \%$ of the trials) phases of the experiment and for the explicit, partially explicit, and implicit instruction groups. Error bars refer to the standard errors of the means, computed across subjects.

ses, error rate was transformed into $z$ scores ( probit transform) to avoid ceiling effects. The average error rate values for all groups and the flanker effects for the explicit and implicit groups are presented in the bottom portion of Figure 1 and in the right panel of Figure 2, respectively (average error rates are presented in Table 1). However, we also present the corresponding mean percentage error rates (not transformed) in Table 1 for ease of comparison with the literature. An overall ANOVA revealed a significant effect of noise type $\left[F(1,79)=383.71, M S_{\mathrm{e}}=0.317\right.$, $p<.0001$ ], with higher accuracy for compatible than for incompatible noise trials (the mean $z$ values correspond to an accuracy level of .974 and .914 for the two noise conditions, respectively). The flanker effect, however, interacted with the prediction cue $\left[F(2,158)=21.11, M S_{\mathrm{e}}=0.074\right.$, $p<.0001]$. The effect of noise was largest in the predictcompatible condition (in which the $z$ scores corresponded to accuracy level of .973 for compatible arrays and of .900 for incompatible arrays), intermediate for the neutral condition (in which the $z$ scores corresponded to an accuracy

Table 1

Average Error Rates (in Percentages) for Each Experimental Condition and Related Standard Errors of the Means Computed Across Subjects

\begin{tabular}{|c|c|c|c|c|c|c|c|}
\hline \multirow[b]{3}{*}{ Instructions } & \multirow[b]{3}{*}{ Trial } & \multicolumn{6}{|c|}{ Cue } \\
\hline & & \multicolumn{2}{|c|}{$\mathrm{PC}$} & \multicolumn{2}{|c|}{$\mathrm{PN}$} & \multicolumn{2}{|c|}{ PI } \\
\hline & & $M$ & SEM & $M$ & SEM & $M$ & $S E M$ \\
\hline \multicolumn{8}{|c|}{ Early Phase } \\
\hline \multirow[t]{2}{*}{ Explicit } & Compatible & 2.37 & 0.38 & 2.09 & 0.42 & 1.70 & 0.51 \\
\hline & Incompatible & 9.60 & 1.59 & 8.19 & 1.07 & 5.69 & 0.72 \\
\hline \multirow[t]{2}{*}{ Partial } & Compatible & 3.21 & 0.69 & 3.42 & 0.85 & 2.44 & 0.76 \\
\hline & Incompatible & 12.80 & 1.95 & 10.90 & 1.16 & 9.11 & 1.03 \\
\hline \multirow[t]{3}{*}{ Implicit } & Compatible & 2.27 & 0.60 & 2.69 & 0.65 & 1.93 & 0.62 \\
\hline & Incompatible & 7.97 & 2.18 & 8.38 & 1.62 & 6.21 & 1.17 \\
\hline & & Mid & Phase & & & & \\
\hline \multirow[t]{2}{*}{ Explicit } & Compatible & 2.14 & 0.41 & 2.01 & 0.32 & 2.42 & 0.47 \\
\hline & Incompatible & 8.66 & 1.53 & 8.40 & 1.29 & 5.28 & 0.74 \\
\hline \multirow[t]{2}{*}{ Partial } & Compatible & 3.25 & 0.74 & 3.18 & 0.77 & 3.01 & 0.76 \\
\hline & Incompatible & 12.18 & 1.55 & 12.24 & 1.88 & 7.43 & 0.98 \\
\hline \multirow[t]{2}{*}{ Implicit } & Compatible & 3.38 & 0.74 & 3.10 & 0.63 & 3.00 & 0.73 \\
\hline & Incompatible & 7.87 & 1.50 & 9.99 & 1.71 & 5.38 & 1.01 \\
\hline \multicolumn{8}{|c|}{ Late Phase } \\
\hline \multirow[t]{2}{*}{ Explicit } & Compatible & 2.02 & 0.37 & 2.20 & 0.55 & 2.32 & 0.65 \\
\hline & Incompatible & 10.14 & 2.15 & 9.64 & 1.30 & 5.26 & 0.95 \\
\hline \multirow[t]{2}{*}{ Partial } & Compatible & 2.93 & 0.59 & 3.05 & 0.72 & 3.12 & 0.86 \\
\hline & Incompatible & 11.25 & 2.37 & 12.06 & 1.65 & 8.22 & 1.11 \\
\hline \multirow[t]{2}{*}{ Implicit } & Compatible & 3.21 & 0.59 & 2.75 & 0.54 & 2.87 & 0.65 \\
\hline & Incompatible & 9.98 & 1.74 & 9.09 & 1.58 & 6.29 & 1.11 \\
\hline
\end{tabular}

Note-PC, predict compatible; PN, predict neutral; PI, predict incompatible. 
level of .973 for compatible arrays and of .902 for incompatible arrays), and smallest in the predict-incompatible condition (in which the $z$ scores corresponded to an accuracy level of .975 for compatible arrays and of .936 for incompatible arrays). In addition to corroborating the RT effects, these data suggest that a speed-accuracy tradeoff could not account for the results. Also consistent with the RT data, there was no effect of the instruction conditions on error rate, neither as a main effect nor in any interaction. In particular, the three-way interaction between instruction, cue, and noise level was not significant $\left[F(4,158)=1.01, M S_{\mathrm{e}}=0.074\right.$, n.s. $]$. As for RTs, to determine whether strategic effects occurred during the late phase for the subjects in the implicit instruction group, we also computed the difference between the flanker effect on accuracy in the predict-compatible and predictincompatible conditions. Again, as for RTs, our hypothesis was directional, since we expected a larger effect for the predict-compatible than for the predict-incompatible condition. This difference was in fact significant $[t(26)=$ $1.94, p<.05$, one-tailed].

Thus, these data indicate that, even without being informed explicitly about the predictive values of the cues, the subjects in the partially explicit and implicit instruction groups performed similarly to the subjects in the explicit instruction group on both RT and accuracy, particularly toward the end of the experiment. When a cue predicted that a compatible trial was more likely to follow, the subjects in all the groups chose a strategy resulting in a large difference between the RT and accuracy obtained on compatible and incompatible trials (a parallel strategy). When a cue predicted that an incompatible trial was more likely to follow, the subjects in all the groups chose a strategy resulting in a smaller difference between the RT and accuracy on compatible and incompatible trials (a focused strategy). Thus, by the end of training, instructions did not appear to influence performance. The data replicate the strategic variations in the flanker effect reported by Gratton et al. (1992) and confirm that the size of the flanker effect can be manipulated by using symbolic cues. They also show that the subjects in all three instructions groups were able to use the strategies in a similar manner, regardless of the instructions they received.

This fact raises the question as to whether the subjects in the partially explicit and implicit groups (who were not explicitly told about the meaning of the cues) were, in fact, aware of their predictive values. To answer this question, two objective tests of explicit knowledge of the cue significance were carried out at the end of the experiment: (1) a probability estimation task and (2) a forced choice task. The forced choice task results are given in Figure 3. The subjects in the explicit instruction group showed that they understood and remembered the instructions that they had been given at the beginning of the experiment. Despite the fact that the cues influenced RT and accuracy for all the groups, the subjects in the implicit instruction group did not indicate awareness of the predictive value of the cues, and the subjects in the partially explicit group showed a reduced effect, as compared with those in the explicit instruction group. The repeated measures ANOVA

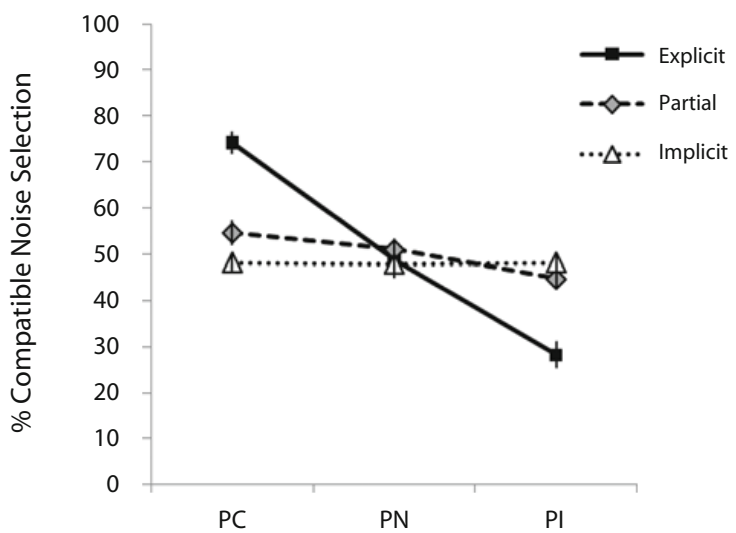

Figure 3. Average percentages of compatible choices in a forced choice task given by the explicitly, partially explicitly, and implicitly instructed subjects given predict-compatible (PC), predictneutral (PN), and predict-incompatible (PI) cues. Error bars refer to the standard errors of the means, computed across subjects.

revealed a main effect of cues $\left[F(2,158)=48.11, M S_{\mathrm{e}}=\right.$ $148.06, p=.0001]$. The instruction $\times$ cue interaction was also significant $\left[F(4,158)=27.49, M S_{\mathrm{e}}=148.06\right.$, $p<.00001]$. Planned comparisons showed significant differences for the explicit instruction group between the predict-compatible and neutral conditions $[F(1,27)=$ 76.97, $p<.0001]$, the predict-compatible and predictincompatible conditions $[F(1,27)=85.19, p<.0001]$, and the neutral and predict-incompatible conditions $[F(1,27)=35.76, p<.0001]$. A significant difference was also found for the partially explicit group between the predict-compatible and predict-incompatible conditions $[F(1,26)=6.85, p<.02]$ and between the neutral and predict-incompatible conditions $[F(1,26)=4.85, p<$ $.05]$. However, no significant differences were found be-

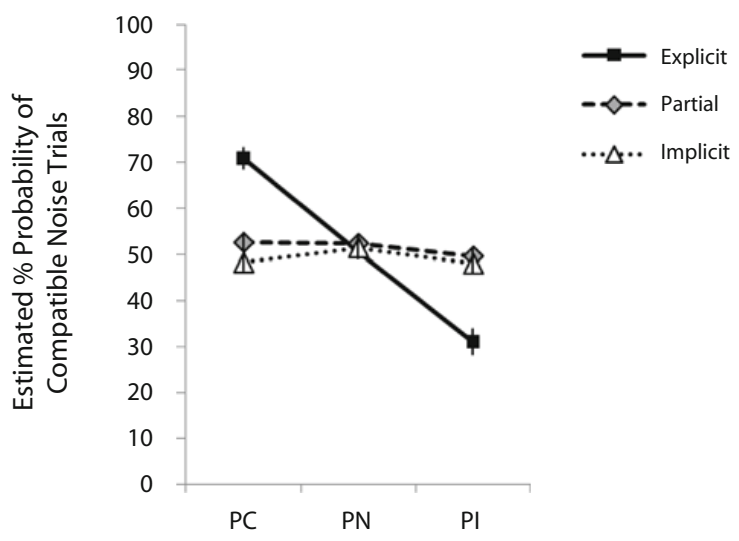

Figure 4. Average percentages for estimates of compatible trial frequency in a probability estimation task given by the explicitly, partially explicitly, and implicitly instructed subjects given predict-compatible (PC), predict-neutral $(\mathrm{PN})$, and predictincompatible (PI) cues. Error bars refer to the standard errors of the means, computed across subjects. 
tween any of the three conditions for the implicit instruction group (all $F s<1$ ).

Figure 4 shows the results for the probability estimation task. In this figure, the average percentages for estimates that a compatible trial would follow a given cue are plotted as a function of the instructions the subjects received. The mixed-design measures ANOVA revealed a main effect of cues $\left[F(2,158)=37.25, M S_{\mathrm{e}}=115.12\right.$, $p<.0001]$. The instruction $\times$ cue interaction was also significant $[F(4,158)=29.57, p<.0001]$. Planned comparisons showed that the only significant differences were found in the explicit group between the predict-compatible and predict-incompatible conditions $[F(1,27)=68.39$, $p<.0001]$, the predict-compatible and neutral conditions $[F(1,27)=53.68, p<.0001]$, and the neutral and predict-incompatible conditions $[F(1,27)=49.99, p<$ $.0001]$. These data show that the subjects in the explicit group were able to use their knowledge about the predictive value of the cues. However, there is little or no evidence that the subjects in the partially explicit or implicit instruction groups were aware of the specific predictive value of the cues.

\section{DISCUSSION}

The results show that subjects can select strategies and use them, even in the absence of explicit instructions and awareness about the meaning of the cues. For example, when the cue predicted that a compatible trial was more likely to follow, the subjects presumably adopted a parallel strategy (Gratton et al., 1992) or did not gate the automatic process (Kunde, 2003). That is, the subjects appear to have relied on evidence accumulated during early, parallel phases of processing, so that their responses on compatible noise trials were fast; however this information was not selective, so that incompatible noise generated large amounts of conflict. In this case, the difference between the RT on compatible and incompatible trials was large. A converse logic applies to the use of the focused/gating strategy, leading to smaller flanker effects.

The present experiment replicated the findings of Gratton et al. (1992), indicating that manipulations designed to affect the subjects' expectancy for compatible and incompatible noise conditions do affect the size of the flanker effect in a manner consistent with the idea that subjects use adaptive response strategies. Strategy selection was presumably based on an analysis of the costs and benefits associated with each choice. The subjects used a strategy that helped them improve performance, depending on the predictive value of the cues. This improvement in performance was due largely to effects on incompatible trials, such that the focused strategy reduced the influence of the flankers on incompatible trials without influencing RTs or errors on compatible trials.

An alternative view to the parallel/focused distinction proposed by Gratton et al. (1992) holds that subjects, when expecting incompatible noise, switch from processing the target to processing the distractors, inverting the stimulusresponse rule (see Wühr \& Kunde, 2008). However, this alternative view is inconsistent with some of the results of the present study. The data show that even when subjects expect incompatible noise, their responses are clearly faster for compatible than for incompatible trials. This would be impossible if the subjects had switched to focus only on the flanker letters and reversed their stimulus-response rule. In fact, in the unpredicted compatible trials, this reverse response strategy would be particularly difficult, since the subjects would have to reverse their response mode a second time (this time, after the presentation of the stimulus) to accurately respond to the target, thus generating a particularly slow response (which, instead, does not occur). It should also be noted that the accuracy for compatible trials does not vary at all across the three cue conditions, a result that is completely inconsistent with the double inversion of the response rule required for these trials. Indeed, there is ample evidence in the literature that the continuous rule switching predicted by this alternative hypothesis would be extremely difficult and fatiguing for human subjects. The work of Logan and Zbrodoff $(1979,1982)$ indicates that instantiation of task rules takes a considerable amount of time $(>500 \mathrm{msec})$, thus making it unlikely that subjects could (and/or would) make these changes on the fly, after the presentation of the imperative stimulus.

Another alternative explanation is that, after a predictincompatible cue (or generally, across the entire study), subjects operate as if in a four-choice, rather than a twochoice, paradigm: They process stimuli globally, not paying attention specifically to the target-noise distinction, but only to the general stimulus appearance. This explanation also fails to account for the fact that compatible trials are faster than incompatible trials, even when their conditional probability is smaller. So both alternative views can be discarded.

The present study extends the analysis of strategic processing carried out by Gratton et al. (1992). In particular, it shows that (1) explicit instructions about the significance of the cue are not required for the occurrence of strategic modulation, since the subjects in the implicit group showed evidence of strategic modulation of performance by the end of the experiment; and (2) it is possible to develop and use optimal performance strategies even in the absence of explicit knowledge about the predictive value of the cues provided in a task. This is demonstrated by the results of the paper-and-pencil tests administered after the conclusion of the flanker task.

To test the subjects' state of awareness, three tests were used in the experiment: two direct measures (probability of estimation and forced choice tasks) and two indirect measures (RT and error rate). The subjects in the implicitly instructed group performed no better than chance on both direct tests. It can be argued that they were not aware of any relations between the cues and the stimulus arrays. Reingold and Merikle (1988) argued that unconscious learning is demonstrated if an indirect measure (such as RT) is more sensitive than a direct measure (such as the forced choice test). Indeed, in the present study, implicitly instructed subjects showed improved performance on RT but performed no better than would be expected by chance on the forced choice tests. Thus, it is reasonable to conclude that the present study elicited implicit learning of strategies. 
Similarly to our study, Crump, Vaquero, and Milliken (2008; Crump \& Milliken, 2009) also showed that awareness is not required for cognitive control and that providing subjects with explicit information about context-specific knowledge did not change the context-specific congruency effect, a supposed instance of cognitive control in Stroop tasks.

However, it is possible that rather than learning a cuetarget relation, the subjects learned that the cue for incompatible trials prompted them to make a slower, more focused response. That is, the subjects could learn an association between the cue and a slow, focused response. The present research does not distinguish between these two possibilities. Also, the possibility of using implicit knowledge to influence strategy selection raises the question of how implicit processes interact with control processes. This would presumably require an in-depth investigation using different intervals between the cue and the imperative stimulus to study the time course of the activity, as well as using more complex manipulations of the information provided by the cue to address the question of the mechanisms involved. Finally, the relative speed of learning in the explicit and implicit conditions deserves further attention. Is explicit knowledge of the contingencies really helpful in this task? Is it actually used? Future research could address these questions.

The main innovation of the present study is the use of paper-and-pencil tests to assess the explicit knowledge that subjects have about the contingencies between cues and noise levels within the flanker task. This allowed us to better determine whether explicit knowledge is a prerequisite for the occurrence of control processes. The evidence is clearly against it. This study sets itself within the context of a long series of studies of control processes in conflict paradigms, derived primarily from the Gratton et al. (1992) study (although this study had its precursors in the work of Logan and Zbrodoff in the late 1970s; Logan \& Zbrodoff, 1979). This and other work has shown that (1) the modulation of the flanker effect is not due solely to response repetition effects (see Experiment 3 in Gratton et al. [1992] and Verbruggen, Notebaert, Liefooghe, \& Vandierendonck [2006] for examples), contradicting earlier conclusions from Mayr, Awh, and Laurey (2003), but is a form of adaptive control; (2) adaptive control can be location and item specific (see Blais, Robidoux, Risko, \& Besner, 2007; Corballis \& Gratton, 2003; see also Jacoby, Lindsay, \& Hessels, 2003; Jacoby, McElree, \& Trainham, 1999; Trainham, Lindsay, \& Jacoby, 1997); (3) adaptive control is related to stimulus rather than response conflict (Notebaert \& Verguts, 2006; Verbruggen et al., 2006); (4) adaptive control in conflict paradigms can be considered in terms of the relative utilization of automatic and controlled processing (Borgmann, Risko, Stolz, \& Besner, 2007), following a gating mechanism; and (5) adaptive control can be conceptualized as a form of Hebbian learning (Verguts \& Notebaert, 2008; see also Braver \& Cohen, 2001).

Although these studies are all relevant to characterizing aspects of adaptive control, they do not define the extent to which explicit knowledge of the expected noise level is necessary for adaptive control to occur. This is a very im- portant theoretical question because it directly impinges on the relationship between attention and control processes, on the one hand, and explicit knowledge, intention, or consciousness, on the other. Although there is considerable evidence that motor patterns can be learned without intention and explicit knowledge, there is much less evidence that the same is true for adaptive control processes. This is particularly the case because the phenomena underlying adaptive control appear to be related to the weighting of different types of information (related to automatic vs. controlled processing), rather than merely to the speed with which different processes occurs (see Borgmann et al., 2007).

Our results lead to seemingly contrasting conclusions, as compared with those in Kunde (2003). In Kunde's study, subliminal primes were not capable of generating strategic adjustments. Our work differs because we used an implicit-learning approach (rather than a subliminal stimulation approach) to assess the possibility of strategic learning outside awareness. The data clearly indicate that supraliminal stimuli can generate strategic learning even though subjects are unable to explicitly specify the relationships between the cues and imperative stimuli.

How can these results be reconciled? Kunde's (2003) work involves a sequential effect, in which adaptive control is reactive, based on the experience of what has happened on the previous trial (the subjective presence or absence of conflict). In the present study, adaptive control is, instead, proactive, based on the expected probabilities of conflict on the upcoming trial (as indicated by a cue). However, this reactive versus proactive control distinction would lead to the somewhat unsatisfactory conclusion that reactive control (which occurs in Kunde's [2003] work and is essentially a bottom-up phenomenon) requires awareness, whereas proactive control (which occurs in our study and is essentially a top-down phenomenon) does not. This appears to be contrary to basic intuition about these two phenomena.

An alternative account is based on the distinction between the conscious experience of the presentation of the cue and awareness of the relationship between cues and imperative stimuli. As is shown by our experiment, the first is possible without the second. However, the converse may not be true: Awareness of the relationship between cues and imperative stimuli seems to depend on the conscious experience of the cue, as was shown by Kunde (2003). Thus, it appears that two different types of awareness are involved here: perceptual awareness (demonstrated in Kunde's [2003] work) and contingency awareness (demonstrated in our work). These two forms of awareness may depend on different levels of processing and, presumably, on different brain mechanisms.

This account is consistent with research on the effects of brain lesions: Medial-temporal cortex damage may lead to a lack of contingency awareness but leave perceptual awareness intact (e.g., Gabrieli, Cohen, \& Corkin, 1988). However, the blindsight phenomenon (e.g., Weiskrantz, 2002) suggests that contingency awareness might occur in the absence of perceptual awareness. Interestingly, this parallel between our data and the brain lesion data would suggest that the type of contingency learning observed in 
our study may not depend on medial-temporal lobe structures. Two types of circuitry could be involved: one based on basal ganglia, which has often been involved in implicit learning (e.g., Packard \& McGaugh, 1992), and another involving lateral frontal structures, as proposed by Cohen and colleagues (e.g., Braver \& Cohen, 2001). Future work involving brain imaging methods and patients with brain lesion may be useful for investigating these possibilities. In conclusion, our findings are not consistent with the claim that response gating is "an intention-mediated strategy" (see Kunde, 2003, p. 198). Instead, it appears that the subjects in the implicit instruction group selected strategies without making an intentional choice (see also Schmidt, Crump, Cheesman, \& Besner, 2007).

\section{AUTHOR NOTE}

The study presented in this article was submitted by R.G. in partial fulfillment of the requirements for the master's degree in psychology to the Graduate School of the University of Missouri-Columbia. Partial support for this article was provided by ONR-MURI Grant N00014-07-1-0903 to A. Kramer, G.G., and M.F. The authors thank A. Bettencourt for her comments on an earlier draft of this article and A. Ramsey, S. Wade, L. Hill, and J. T. Thatcher for their assistance with data collection. Address all correspondence to T. R. Schachtman, Department of Psychological Sciences, University of Missouri, 210 McAlester Hall, Columbia, MO 65211 (e-mail: schachtmant@missouri.edu).

Note-Accepted by the previous editorial team, when Brian H. Ross was Editor.

\section{REFERENCES}

Blais, C., Robidoux, S., Risko, E. F., \& Besner, D. (2007). Itemspecific adaptation and the conflict-monitoring hypothesis: A computational model. Psychological Review, 114, 1076-1086.

Borgmann, K. W. U., Risko, E. F., Stolz, J. A., \& Besner, D. (2007). Simon says: Reliability and the role of working memory and attentional control in the Simon task. Psychonomic Bulletin \& Review, 14, 313-319.

Braver, T. S., \& CohEn, J. D. (2001). Working memory, cognitive control, and the prefrontal cortex: Computational and empirical studies. Cognitive Processing, 2, 25-55.

Carlson, K. A., \& Flowers, J. H. (1996). Intentional versus unintentional use of contingencies between perceptual events. Perception \& Psychophysics, 58, 460-470.

Cheesman, J., \& Merikle, P. M. (1984). Priming with and without awareness. Perception \& Psychophysics, 36, 387-395.

Coles, M. G. H., Gratton, G., Bashore, T. R., Eriksen, C. W., \& Donchin, E. (1985). A psychophysiological investigation of the continuous flow model of human information processing. Journal of Experimental Psychology: Human Perception \& Performance, 11, 529-553.

Corballis, P. M., \& Gratton, G. (2003). Independent control of processing strategies for different locations in the visual field. Biological Psychology, 64, 191-209.

Crump, M. J. C., \& Milliken, B. (2009). The flexibility of contextspecific control: Evidence for context-driven generalization of itemspecific control settings. Quarterly Journal of Experimental Psychology, 62, 1523-1532.

Crump, M. J. C., Vaquero, J. M., \& Milliken, B. (2008). Contextspecific learning and control: The roles of awareness, task relevance, and relative salience. Consciousness \& Cognition, 17, 22-36.

ERIKSEN, B. A., \& ERIKSEN, C. W. (1974). Effects of noise letters upon the identification of a target letter in a nonsearch task. Perception \& Psychophysics, 16, 143-149.

Gabrieli, J. D. E., Cohen, N. J., \& Corkin, S. (1988). The impaired learning of semantic knowledge following bilateral medial temporallobe resection. Brain \& Cognition, 7, 157-177.

Gratton, G., Coles, M. G. H., \& Donchin, E. (1992). Optimizing the use of information: Strategic control of activation of responses. Journal of Experimental Psychology: General, 121, 480-506.

Gratton, G., Coles, M. G. H., Sirevaha, E. J., Eriksen, C. W., \& Donchin, E. (1988). Pre- and poststimulus activation of response channels: A psychophysiological analysis. Journal of Experimental Psychology: Human Perception \& Performance, 14, 331-344.

JaCoby, L. L., Lindsay, D. S., \& Hessels, S. (2003). Item-specific control of automatic processes: Stroop process dissociations. Psychonomic Bulletin \& Review, 10, 638-644.

Jacoby, L. L., McElree, B., \& Trainham, T. N. (1999). Automatic influences as accessibility bias in memory and Stroop tasks: Toward a formal model. In D. Gopher \& A. Koriat (Eds.), Attention and performance XVII: Cognitive regulation of performance. Interaction of theory and application (pp. 461-486). Cambridge, MA: MIT Press.

KundE, W. (2003). Sequential modulations of stimulus-response correspondence effects depend on awareness of response conflict. Psychonomic Bulletin \& Review, 10, 198-205.

KUNDE, W. (2004). Response priming by supraliminal and subliminal action effects. Psychological Research, 68, 91-96.

KUNDE, W., \& WÜHR, P. (2006). Sequential modulations of corresponding effects across spatial dimensions and tasks. Memory \& Cognition, 34, 356-367.

Logan, G. D., \& ZBRodoff, N. J. (1979). When it helps to be misled: Facilitative effects of increasing the frequency of conflicting stimuli in a Stroop-like task. Memory \& Cognition, 7, 166-174.

Logan, G. D., \& ZBrodoff, N. J. (1982). Constraints on strategy construction in a speeded discrimination task. Journal of Experimental Psychology: Human Perception \& Performance, 8, 502-520.

Mayr, U., Awh, E., \& LaUReY, P. (2003). Conflict adaptation effects in the absence of executive control. Nature Neuroscience, 6, 450-452.

MilLER, J. (1987). Evidence of preliminary response preparation from a divided attention task. Journal of Experimental Psychology: Human Perception \& Performance, 13, 425-434.

NäÄTÄNEN, R. (1982). Processing negativity: An evoked-potential reflection. Psychological Bulletin, 92, 605-640.

Notebaert, W., \& Verguts, T. (2006). Stimulus conflict predicts conflict adaptation in a numerical flanker task. Psychonomic Bulletin \& Review, 13, 1078-1084.

PaCKard, M. G., \& McGaugh, J. L. (1992). Double dissociation of fornix and caudate nucleus lesions on acquisition of two water maze tasks: Further evidence for multiple memory systems. Behavioral Neuroscience, 106, 439-446.

Reingold, E. M., \& Merikle, P. M. (1988). Using direct and indirect measures to study perception without awareness. Perception \& Psychophysics, 44, 563-575.

Schmidt, J. R., Crump, M. J. C., Cheesman, J., \& Besner, D. (2007). Contingency learning without awareness: Evidence for implicit control. Consciousness \& Cognition, 16, 421-435.

Stadler, M. A., Warren, J. L., \& LeSch, S. L. (2000). Is there crossformat transfer in implicit invariance learning? Quarterly Journal of Experimental Psychology, 53A, 235-245.

SZYMANSKI, K. F., \& MaCLeOD, C. M. (1996). Manipulation of attention at study affects an explicit but not an implicit test of memory. Consciousness \& Cognition, 5, 165-175.

Trainham, T. N., LindSAY, D. S., \& Jacoby, L. L. (1997). Stroop process dissociations: Reply to Hillstrom and Logan (1997). Journal of Experimental Psychology: Human Perception \& Performance, 23, $1579-1587$.

Verbruggen, F., Notebaert, W., Liefooghe, B., \& VandierenDONCK, A. (2006). Stimulus- and response-conflict-induced cognitive control in the flanker task. Psychonomic Bulletin \& Review, 13, 328-333.

Verguts, T., \& Notebaert, W. (2008). Hebbian learning of cognitive control: Dealing with specific and nonspecific adaptation. Psychological Review, 115, 518-525.

Weiskrantz, L. (2002). Prime-sight and blindsight. Consciousness \& Cognition, 11, 568-581.

WÜHR, P., \& KUNDE, W. (2008). Precueing spatial S-R correspondence: Is there regulation of expected response conflict? Journal of Experimental Psychology: Human Perception \& Performance, 34, 872-883.

(Manuscript received November 14, 2008; revision accepted for publication October 14, 2009.) 